\title{
Malaria knowledge, preventive actions, and treatment-seeking behavior among ethnic minorities in Ratanakiri Province, Cambodia: a community-based cross-sectional survey
}

\author{
Junko Yasuoka ${ }^{1 *}$ (D), Kimiyo Kikuchi ${ }^{2}$, Keiko Nanishi ${ }^{3}$, Po Ly ${ }^{4}$, Boukheng Thavrin ${ }^{4}$, Tsutomu Omatsu ${ }^{1}$
} and Tetsuya Mizutani ${ }^{1}$

\begin{abstract}
Background: Malaria incidence has been steadily declining in Cambodia, where the government is aiming to eliminate malaria by 2025. Successful malaria elimination requires active engagement and participation of communities to recognize malaria symptoms and the development of prompt treatment-seeking behavior for early diagnosis and appropriate treatment. This study examined malaria knowledge, preventive actions, and treatment-seeking behavior among different groups of ethnic minorities and Khmer in Ratanakiri Province, Cambodia.

Methods: Face-to-face interviews were conducted in December 2015, targeting 388 mothers with children under 2 years old, who belonged to ten ethnic minority groups or the Khmer group living in 62 rural villages in Ratanakiri. In addition to describing mothers' knowledge and actions for malaria prevention, logistic regression analysis was performed to identify determinants of fever during the most recent pregnancy and among children under two.

Results: Overall 388 mothers were identified for enrollment into the study of which 377 (97.2\%) were included in analyses. The majority of mothers slept under bed nets at home (95.8\%) and wore long-sleeved clothes (83.8\%) for malaria prevention. However, knowledge of malaria was limited: $44.6 \%$ were aware of malaria symptoms, $40.6 \%$ knew the malaria transmission route precisely, and 29.2\% knew of mosquito breeding places. Staying overnight at a farm hut was significantly associated with having fever during the most recent pregnancy (adjusted odds ratio [AOR] 2.008, 95\% confidence interval [Cl]: 1.215-3.321) and a child having fever (AOR 3.681, 95\% Cl 1.943-6.972). Mothers' partaking in a variety of malaria preventive actions was protective against fever in children (AOR 0.292, 95\% Cl: 0.136-0. 650). Among those who had fever during pregnancy, 39.4\% did not seek treatment.

Conclusion: Although the majority of mothers took malaria preventive actions, knowledge of malaria epidemiology and vector ecology and treatment-seeking behavior for fever were limited. Staying overnight at farm huts, regardless of the differences in socio-demographic and socio-cultural characteristics, was strongly associated with fever episodes during pregnancy and childhood. This study indicates the necessity of spreading accurate malaria knowledge, raising awareness of health risks related to agricultural practices, and promoting treatment-seeking behavior among ethnic minorities to strengthen their engagement in malaria elimination.
\end{abstract}

Keywords: Malaria, Knowledge, Preventive actions, Treatment-seeking behavior, Ethnic minorities, Cambodia

* Correspondence: jyasuoka@post.harvard.edu

${ }^{1}$ Research and Education Center for Prevention of Global Infectious Diseases

of Animals, Tokyo University of Agriculture and Technology, 3-5-8 Saiwai-cho,

Fuchu-shi, Tokyo 183-8508, Japan

Full list of author information is available at the end of the article

(c) The Author(s). 2018 Open Access This article is distributed under the terms of the Creative Commons Attribution 4.0 International License (http://creativecommons.org/licenses/by/4.0/) which permits unrestricted use, distribution, and reproduction in any medium, provided you give appropriate credit to the original author(s) and the source, provide a link to the Creative Commons license, and indicate if changes were made. The Creative Commons Public Domain Dedication waiver (http://creativecommons.org/publicdomain/zero/1.0/) applies to the data made available in this article, unless otherwise stated. 


\section{Background}

Malaria incidence and mortality have steadily declined in the Greater Mekong sub-region over the past decades [1]. In Cambodia, a marked decrease in Plasmodium falciparum ( $P$. falciparum) has been observed since 2009; this coincides with the scale-up of the distribution of insecticide treated bed nets and the strengthening of Village Malaria Workers (VMWs) who provide early diagnosis and treatment of malaria for their villagers. The number of malaria cases has significantly decreased especially in western Cambodia (i.e. Pailin and Battambang Provinces) [2]. Given the successes observed in the malaria program, the Cambodian government is aiming to eliminate malaria by 2025 [3].

Although malaria transmission in Cambodia has been low recently, 21 of the 25 provinces are still endemic, and more than half of the population (approximately 8.6 million people) is still at risk of malaria. The risk of malaria transmission is the highest in the forest or forest fringe areas in the northeastern part of the country [4]. Although, four provinces in northeastern Cambodia (Rattanakiri, Mondulkiri, Steung Treng, and Kratie Provinces) have only $3 \%$ of the national population, they were reported to have had $43 \%$ and $25 \%$ of falciparum and vivax malaria cases in the country in 2013, respectively [2].

Ratanakiri Province consists mainly of rural, agricultural villages and has one of the lowest socioeconomic standards in the country [4]. Several studies have been conducted to examine the complexity of malaria epidemiology in the Province [5-7]. However, few studies have addressed the behavioral and social aspects of malaria. Therefore, little is known about malaria knowledge, preventive actions, and treatment-seeking behavior of ethnic minorities living in the Province.

In the elimination phase of malaria, complete termination of local transmission is required, especially in areas with foci of malaria transmission, which can remain as a source of infection [5]. In addition to appropriate infrastructure and sufficient resources at primary health care facilities, active engagement of communities to recognize malaria symptoms and take prompt treatment-seeking actions is crucial to reduce the reservoir of malaria parasites [8]. Therefore, communities need to be encouraged to take immediate actions to treat fever in order to promote early diagnosis and appropriate treatment of malaria [9]. Moreover, community knowledge, attitudes and practices should be examined to further promote proper malaria preventive actions and to discourage risk behavior among different ethnic minority groups.

The aim of this study was to examine malaria knowledge, preventive actions, and treatment-seeking behavior among ten ethnic minority groups and the Khmer group in Ratanakiri Province. Additionally, we aimed to identify risk factors for fever during the most recent pregnancy and among children under two. Results from this study will contribute to the development and implementation of future intervention programs to achieve malaria elimination in Cambodia.

\section{Methods \\ Study design}

This study was conducted as a baseline survey for an intervention study, which is currently carried out to integrate community-based malaria control and Maternal, Neonatal, and Child Health (MNCH) services in the Ratanakiri Province, Cambodia. Data were collected from December 23 to 30, 2015, through face-to-face interviews with mothers with children under 2 years of age living in $62 \mathrm{VMW}$ villages.

\section{Study site}

This study was conducted in Ratanakiri Province in the north-eastern part of the country, which shares its borders with Lao People's Democratic Republic (PDR) and Vietnam. Residents consist of 13 ethnic minority groups and the Khmer group, which is the major ethnic group in Cambodia. People generally live in villages which consist of 20-60 families and mainly engage in subsistence shifting agriculture, rice farming, and plantation of rubber, cashew, and cassava. As the majority of ethnic minority groups live on subsistence slash-and-burn farming and rice cultivation, many of them own farm huts (plot huts) near or inside the forest, where they stay to plant, guard, and harvest crops [10,11].

Out of 27 health centers in Ratanakiri province, 12 that cover villages where Village Malaria Workers (VMWs) are engaged in malaria control activities, were included in this study (VMW villages). Seven out of the 12 health centers were then selected based on accessibility by vehicles or motorcycles throughout the year (purposive sampling). These included Andaung Meas, Chomrom Bay Sruk, Kachanh, Lumphat, Ochum, Oyadav, and Taveng. Of the $73 \mathrm{VMW}$ villages serviced by the seven health centers, ten villages under the Ochum health center and one village under the Andaung Meas health center were excluded, considering the geographical difficulty in conducting a long-term intervention program scheduled after the survey, and absence of eligible interviewees at the time of the survey. Therefore, in total 62 VMW villages were selected for inclusion in this study.

\section{Data collection}

\section{Survey data}

A semi-structured questionnaire was developed to conduct the face-to-face interviews. Most of the questions were drawn from existing questionnaires, including the Cambodia Demographic Health Survey 2014 [12] and 
questionnaires that were already used in Cambodia for previous studies on VMWs and mothers with children under five [13-15]. The questionnaire contained questions regarding a) mothers' and their families' socio-demographic characteristics; b) agricultural practices; c) history of fever and infectious diseases (malaria, diarrhea, and pneumonia, etc.); d) knowledge and actions for malaria prevention; e) treatment-seeking behavior of mothers for themselves and their children under two; f) mothers' complications during pregnancy and childbirth (fever, anemia, bleeding, etc.); and g) children's complications (fever, difficulty breathing, etc.). The questionnaire was developed in English, translated into Khmer by Cambodian health experts, and then verified by other Khmer health experts to ensure the accuracy of the translation.

Indices to measure mothers' knowledge on malaria epidemiology and vector ecology and actions for malaria prevention and vector control were developed based on mothers' answers to the survey questions, as described in previous publications [13, 16]. Knowledge indices measured to quantify understanding of malaria epidemiology and vector ecology included: malaria symptoms, malaria transmission, vector species, vector active time, vector development time, breeding places, and natural enemies. Regarding malaria symptoms, mothers were asked if the following are correct: stomach ache, diarrhoea, nausea, fever, and shivering. Choices given to answer malaria transmission included cough or sneeze, touching blood, touching utensils, sharing food, coming close to mosquitoes, and mosquito bites. Choices on mosquito genera and sex were given for vector species. For vector's most active time, the following time periods were given, morning, afternoon, and dusk to dawn. Vector development time was asked by an open-ended question. Regarding vector breeding places, trees, on the ground, and water pools around houses, water pools in the forest were given. For natural enemies of the vector, four choices were given: dogs, birds, aquatic insects, and small fish. Correct answer for each item was given one point.

Action indices were developed to quantify different malaria preventive and vector control measures taken by mothers. Each measure that mothers undertook was given one or two points, according to its effectiveness and frequency $[13,16]$. A maximum of two points were given to effective measures (always/most of the time $=2$, sometimes/rarely $=1$, never $=0$ ), including "come back home before dusk," "wear long-sleeved shirts/pants", "sleep under bed nets at home", "refrain from going to the forest", "bring hammock nets to the forest", "fill in water pools" "burn trash around house", "seal holes/ cracks on walls/ceilings", and "cover water jars/tanks". A maximum of one point (always/most of the time/sometimes $=1$, rarely/never $=0$ ) was given to less effective measures including "kill mosquitoes by hands", "use mosquito coils", "spray house", and "clear bush around house". For a wrong measure "plant flowers/grasses around house", 0 points (always/most of the time/sometimes $/$ rarely $=0$, never $=1$ ) were given. The total points for five malaria preventive measures and nine vector control measures became index scores, ranging from 0 to 10 and 0 to 13 , respectively. Using each index, study population was divided into tertiles, namely least active, active, and most active groups.

Seventeen surveyors visited each participant's residence and conducted face-to-face interviews. Before conducting the interviews, malaria experts of the National Center for Parasitology, Entomology, and Malaria Control, Cambodia (CNM) and lead researchers conducted a one-day training for the surveyors to explain each question in the questionnaire and how to conduct the interviews.

We recruited all mothers with children under two residing in 62 VMW villages, who were available at the time of the survey, in order to capture malaria knowledge, preventive actions, and treatment-seeking behavior of mothers in the study site as accurately as possible. Although 388 mothers were interviewed, 11 were excluded from analysis because of missing data. In total, 377 mothers were included in the analysis.

\section{GPS data collection and analysis}

GPS data were collected to measure actual travel distance between the 62 villages and their nearest health centers. Four GPS data collectors traveled from each village to its nearest health center with Holux m-241 (a wireless GPS logger). GPS data were recorded every 5 seconds during each trip. Data were entered into Excel 2016 through the Holux exTour for Logger v2.1 software, and then into Arc Map 10.4, which was used to calculate the actual distance traveled.

\section{Data management and statistical analysis}

All data were coded, entered into data analysis software, and double-checked by the authors to ensure accuracy. Data analysis was done by STATA version 14. Descriptive analyses were conducted to describe sociodemographic characteristics, knowledge and actions for malaria prevention, and treatment-seeking behavior for fever management among participants. Mothers were classified into socio-economic status quartiles based on household assets and housing characteristics determined using a principal component analysis $[15,17]$.

Basic descriptive statistics such as frequency and percentages were used to describe mothers with correct knowledge on malaria epidemiology and vector ecology as well as those who took each measure for malaria prevention and vector control. Knowledge and action 
indices were compared between mothers who had fever and those who did not have fever during the most recent pregnancy as well as between mothers with a child who had fever and those with a child who did not have fever during the last 3 months.

To identify determinants of fever during the most recent pregnancy and fever among their children less than 2 years of age, two multiple logistic regression analyses were conducted. Outcomes of these analyses were reported occurrence of fever during pregnancy and that of fever among children during the last 3 months. Also, the following independent variables were included: mother's age, education, wealth quartiles, ethnicity, having other children or not, age and gender of the child, experience of child loss, whether water was treated prior to consumption, whether there was a toilet at home, whether the family owned livestock, indices to describe malaria preventive measures and vector control measures undertaken, and whether the mother had an agricultural practice which required staying overnight at a farm hut.

Of the sociodemographic characteristics investigated, literacy was excluded from the analysis because of multicollinearity with education $(r=0.89)$. Mother's marital status and occupation were excluded because of the small sub-group sample sizes (most of the mothers were married and were farmers). Child age and child gender were also excluded from the analysis for fever during pregnancy because they were unknown factors during pregnancy. $P$-values $<0.05$ were considered statistically significant.

\section{Results}

Sociodemographic characteristics of 377 mothers with children under two living in $62 \mathrm{VMW}$ villages are described in Table 1 . The majority of the mothers $(72.1 \%)$ were under thirty, most were married $(97.6 \%$, data not shown) and were farmers (91.3\%, data not shown), and two thirds $(68.2 \%)$ responded that they were illiterate. Most of the mothers were farmers $(91.3 \%$, data not shown). This study included mothers who belonged to the Khmer ethnic group (11.1\%) and ten other ethnic minority groups, mainly Thompoun (23.3\%), Charay (20.2\%), and Kreung (15.4\%). Approximately a tenth (11.1\%) of the mothers had experienced stillbirth, and $13.0 \%$ had lost children in the past.

Mothers' knowledge and action for malaria prevention and vector control is summarized in Table 2. Less than half of the mothers were aware of malaria symptoms and transmission route ( $44.6 \%$ and $40.6 \%$, respectively). The majority $(78.0 \%)$ of the mothers recognized the time when mosquitoes are most active. Regarding other aspects of mosquito ecology, including breeding places, species, natural enemies, and time to develop from egg to adult stage, mothers' knowledge was scarce (29.2\%,
$23.9 \%, 19.1 \%$, and $3.4 \%$, respectively). The majority of the mothers used bed nets at home (95.8\%) and wore long-sleeved shirts/pants (83.8\%) to prevent malaria. Approximately two thirds $(68.7 \%)$ reported that they bring hammock nets to the forest. To control mosquitoes, approximately half of the mothers reported that they fill in stagnant water pools (50.1\%), kill mosquitoes using their hands (48.5\%), cover water jars/tanks (46.2\%), burn trash around house (46.2\%), and clear bush around house (40.8\%).

Indices to measure knowledge and action for malaria prevention and vector control were compared between mothers with and without fever during the most recent pregnancy and between mothers' children with and without fever during the last 3 months (Table 3). Mothers who didn't have fever during pregnancy had significantly greater knowledge about malaria symptoms compared to those who had fever during pregnancy $(0.478$ vs 0.354 , $P=0.032$ ). Mothers whose child did not have fever during the last 3 months had significantly greater knowledge about malaria symptoms $(0.482$ vs $0.273, P=$ $0.002)$, mosquito breeding places (0.585 vs $0.439, P=$ $0.030)$, and mosquitoes' natural enemies ( 0.469 vs 0.258 , $\mathrm{P}=0.002)$ compared to mothers whose child had fever during the last 3 months. Additionally, a greater variety of malaria preventive measures were taken among mothers whose child didn't have fever compared to those whose child had fever during the last 3 months (7.640 vs $6.636, P<0.001$ ).

Factors associated with having fever during the most recent pregnancy and children having fever during the last 3 months are described in Table 4. Mothers who had an agricultural practice which required them to stay overnight at a farm hut were approximately twice as likely to have had fever during pregnancy, compared to those who don't stay at a farm hut (adjusted odds ratio [AOR] 2.008, 95\% confidence interval [CI]: 1.215-3.321). Children of mothers who stayed at a farm hut were 3.7 times more likely to have had fever during the last 3 months compared to those whose mothers don't stay at a farm hut (AOR 3.681, 95\% CI: 1.943-6.972). Additionally, children of mothers who were most active to prevent malaria were approximately $70 \%$ less likely to experience fever during the last 3 months compared to children with mothers who were less active in terms of malaria prevention (AOR 0.292, 95\% CI: 0.136-0.650).

Treatment-seeking behavior for fever management is summarized in Table 5. Among 377 study participants, 99 (26.3\%) mothers had fever during the most recent pregnancy. Nearly half of them sought treatment from public providers: approximately $37.4 \%$ from the health center and $12.1 \%$ from VMWs. Approximately $40 \%$ of mothers did not seek treatment. Among those who sought treatment $(n=60)$, more than half $(57.9 \%)$ sought 
Table 1 Sociodemographic characteristics of the study population, stratified by the occurrence of fever during pregnancy and fever of children during the last 3 months (Total $n=377$ in 62 villages)

\begin{tabular}{|c|c|c|c|c|c|c|c|c|}
\hline \multirow[t]{2}{*}{ Characteristics } & \multicolumn{2}{|c|}{ Overall $(n=377)$} & \multicolumn{2}{|c|}{ Fever in pregnancy $(n=99)$} & \multirow[t]{2}{*}{$p$-value } & \multicolumn{2}{|c|}{ Child had fever $(n=66)$} & \multirow[t]{2}{*}{$p$-value } \\
\hline & $\bar{n}$ & $\%$ & $\bar{n}$ & $\%$ & & $\mathrm{n}$ & $\%$ & \\
\hline \multicolumn{9}{|l|}{ Age } \\
\hline$<20$ & 80 & 21.2 & 26 & 26.3 & 0.165 & 16 & 24.2 & 0.622 \\
\hline $20-29$ & 192 & 50.9 & 50 & 50.5 & & 32 & 48.5 & \\
\hline $30-39$ & 89 & 23.6 & 22 & 22.2 & & 17 & 25.8 & \\
\hline $40+$ & 16 & 4.2 & 1 & 1.0 & & 1 & 1.5 & \\
\hline \multicolumn{9}{|l|}{ Education } \\
\hline None & 251 & 66.6 & 65 & 65.7 & 0.795 & 45 & 68.2 & 0.975 \\
\hline Primary & 98 & 26.0 & 28 & 28.3 & & 17 & 25.8 & \\
\hline Secondary/above & 28 & 7.4 & 6 & 6.1 & & 4 & 6.1 & \\
\hline \multicolumn{9}{|l|}{ Literacy } \\
\hline Cannot read at all & 257 & 68.2 & 69 & 69.7 & 0.237 & 48 & 72.7 & 0.661 \\
\hline Able to read only part & 100 & 26.5 & 28 & 28.3 & & 16 & 24.2 & \\
\hline Able to read whole & 20 & 5.3 & 2 & 2.0 & & 2 & 3.0 & \\
\hline \multicolumn{9}{|l|}{ Husband's education } \\
\hline None & 170 & 45.1 & 42 & 42.4 & 0.753 & 32 & 48.5 & 0.333 \\
\hline Primary & 137 & 36.3 & 39 & 39.4 & & 26 & 39.4 & \\
\hline Secondary and above & 70 & 18.6 & 18 & 18.2 & & 8 & 12.1 & \\
\hline Stay overnight at farm hut & 188 & 49.9 & 62 & 62.6 & 0.003 & 48 & 72.7 & $<0.001$ \\
\hline \multicolumn{9}{|l|}{ Wealth quartiles } \\
\hline Low & 95 & 25.2 & 27 & 27.3 & 0.862 & 19 & 28.8 & 0.721 \\
\hline Lower middle & 94 & 24.9 & 24 & 24.2 & & 17 & 25.8 & \\
\hline Upper middle & 94 & 24.9 & 26 & 26.3 & & 13 & 19.7 & \\
\hline High & 94 & 24.9 & 22 & 22.2 & & 17 & 25.8 & \\
\hline \multicolumn{9}{|l|}{ Ethnicity } \\
\hline Khmer & 42 & 11.1 & 8 & 8.1 & 0.053 & 3 & 4.5 & 0.355 \\
\hline Thompoun & 88 & 23.3 & 28 & 28.3 & & 19 & 28.8 & \\
\hline Charay & 76 & 20.2 & 14 & 14.1 & & 14 & 21.2 & \\
\hline Kreung & 58 & 15.4 & 22 & 22.2 & & 10 & 15.2 & \\
\hline Other & 113 & 30.0 & 27 & 27.3 & & 20 & 30.3 & \\
\hline \multicolumn{9}{|l|}{ Number of children } \\
\hline 1 & 140 & 37.1 & 42 & 42.4 & 0.365 & 25 & 37.9 & 0.796 \\
\hline 2 & 112 & 29.7 & 23 & 23.2 & & 17 & 25.8 & \\
\hline $3-4$ & 99 & 26.3 & 26 & 26.3 & & 18 & 27.3 & \\
\hline $5+$ & 26 & 6.9 & 8 & 8.1 & & 6 & 9.1 & \\
\hline \multicolumn{9}{|l|}{ Child age (month) } \\
\hline $0-5$ & 141 & 37.4 & - & - & N/A & 22 & 33.3 & 0.469 \\
\hline $6-11$ & 96 & 25.5 & - & - & & 21 & 31.8 & \\
\hline $12-17$ & 73 & 19.4 & - & - & & 14 & 21.2 & \\
\hline $18-23$ & 67 & 17.8 & - & - & & 9 & 13.6 & \\
\hline Child gender (female) & 181 & 48.0 & - & - & N/A & 36 & 54.5 & 0.242 \\
\hline Experience of stillbirth (Yes) & 42 & 11.1 & 12 & 12.1 & 0.718 & 10 & 15.2 & 0.254 \\
\hline Experience of child loss (Yes) & 49 & 13.0 & 13 & 13.1 & 0.963 & 13 & 19.7 & 0.075 \\
\hline
\end{tabular}


Table 1 Sociodemographic characteristics of the study population, stratified by the occurrence of fever during pregnancy and fever of children during the last 3 months (Total $n=377$ in 62 villages) (Continued)

\begin{tabular}{|c|c|c|c|c|c|c|c|c|}
\hline \multirow[t]{2}{*}{ Characteristics } & \multicolumn{2}{|c|}{ Overall $(n=377)$} & \multicolumn{2}{|c|}{ Fever in pregnancy $(n=99)$} & \multirow[t]{2}{*}{$p$-value } & \multicolumn{2}{|c|}{ Child had fever $(n=66)$} & \multirow[t]{2}{*}{$p$-value } \\
\hline & $n$ & $\%$ & $n$ & $\%$ & & $n$ & $\%$ & \\
\hline \multicolumn{9}{|c|}{ Actual travel distance to health center $(\mathrm{km})$} \\
\hline$<5.0$ & 42 & 11.1 & 8 & 8.1 & 0.663 & 10 & 15.2 & 0.432 \\
\hline $5.0-9.9$ & 98 & 26.0 & 26 & 26.3 & & 18 & 27.3 & \\
\hline $10.0-14.9$ & 132 & 35.0 & 38 & 38.4 & & 18 & 27.3 & \\
\hline $15.0+$ & 105 & 27.9 & 27 & 27.3 & & 20 & 30.3 & \\
\hline Water treatment (Yes) & 222 & 58.9 & 64 & 64.6 & 0.175 & 39 & 59.1 & 0.970 \\
\hline Have toilet (Yes) & 73 & 19.4 & 19 & 19.2 & 0.960 & 15 & 22.7 & 0.446 \\
\hline Own livestock (Yes) & 257 & 68.2 & 69 & 69.7 & 0.704 & 43 & 65.2 & 0.562 \\
\hline
\end{tabular}

Marital status and occupation ommitted (Almost all are married and farmers)

Table 2 Knowledge and action for malaria prevention and vector control among the study population

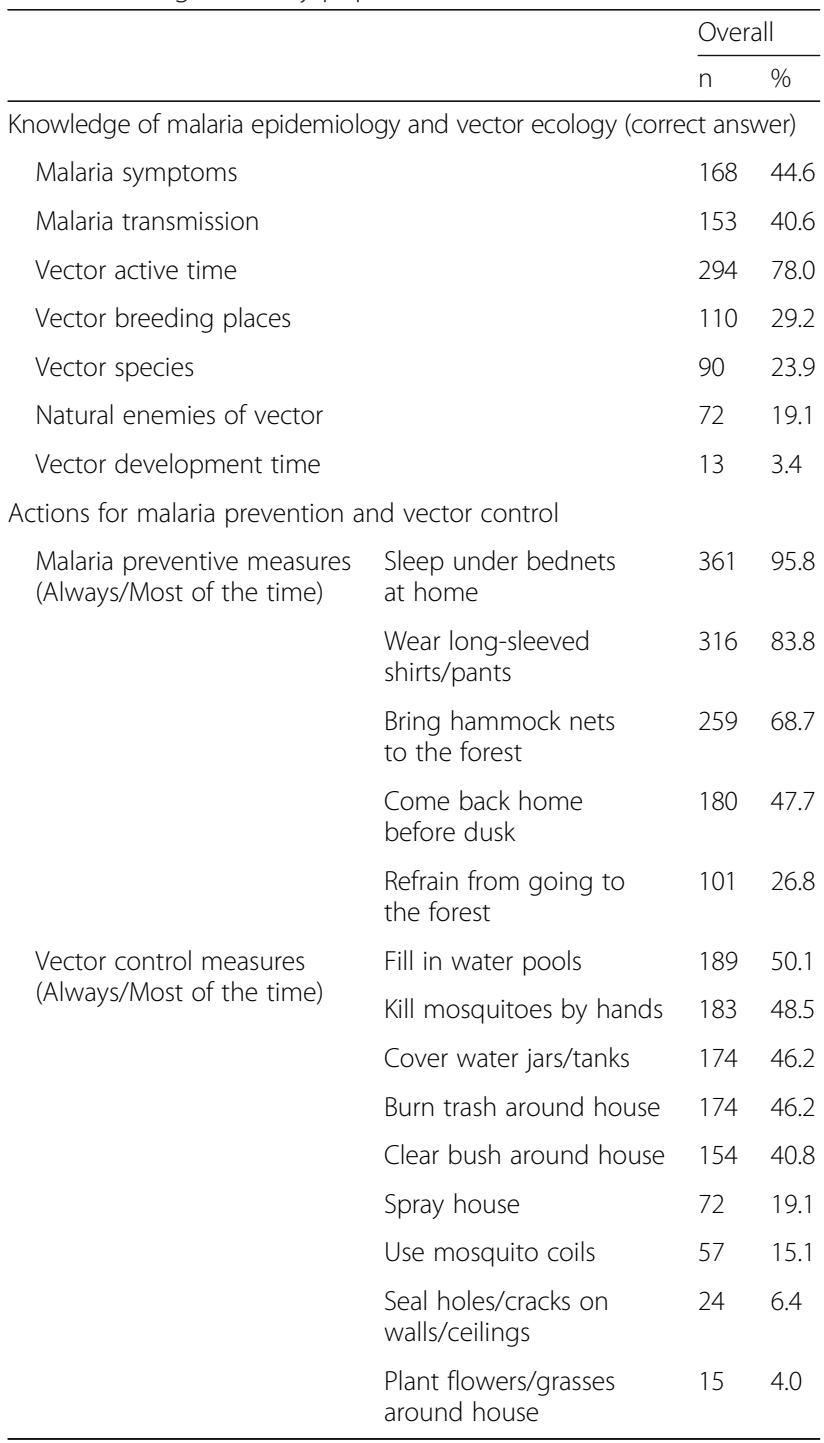

treatment the same day or the day following the onset of fever. Approximately $60 \%$ were sure that they received a blood test for malaria diagnosis, and five of them were found to be positive (three P. falciparum cases and two P. vivax cases).

Sixty-five mothers (17.2\%) reported that their child had fever during the last 3 months. Nearly $60 \%$ of them sought treatment from public providers: about half (47.0\%) from health centers and $12.1 \%$ from VMWs. About one out of seven mothers (13.6\%) did not seek treatment. Out of mothers who sought treatment $(n=$ $57)$, majority of them did so on the same day (31.6\%) or the next day (49.1\%) from the onset of fever. Less than half $(42.2 \%)$ were sure that their child received blood test, and three reported positive results (two P. falciparum cases and one $P$. vivax case).

\section{Discussion}

To our knowledge, this is the first study which reported knowledge and preventive actions for malaria and treatment-seeking behavior for fever among mothers with children under two in Ratanakiri Province, Cambodia. Although the majority of mothers used bed nets at home and wore long-sleeved clothes to prevent malaria, more than half of the mothers were not aware of malaria symptoms and transmission route. Staying overnight at a farm hut was significantly associated with having fever during pregnancy as well as a child having fever during the last 3 months. Mothers' undertaking a variety of malaria preventive actions was found to be a protective factor against the development of fever among children. Our findings suggest that improvements are needed in treatment-seeking behavior as nearly half of the mothers did not seek treatment for fever during pregnancy, and one in seven mothers did not seek treatment when their child had a fever. 


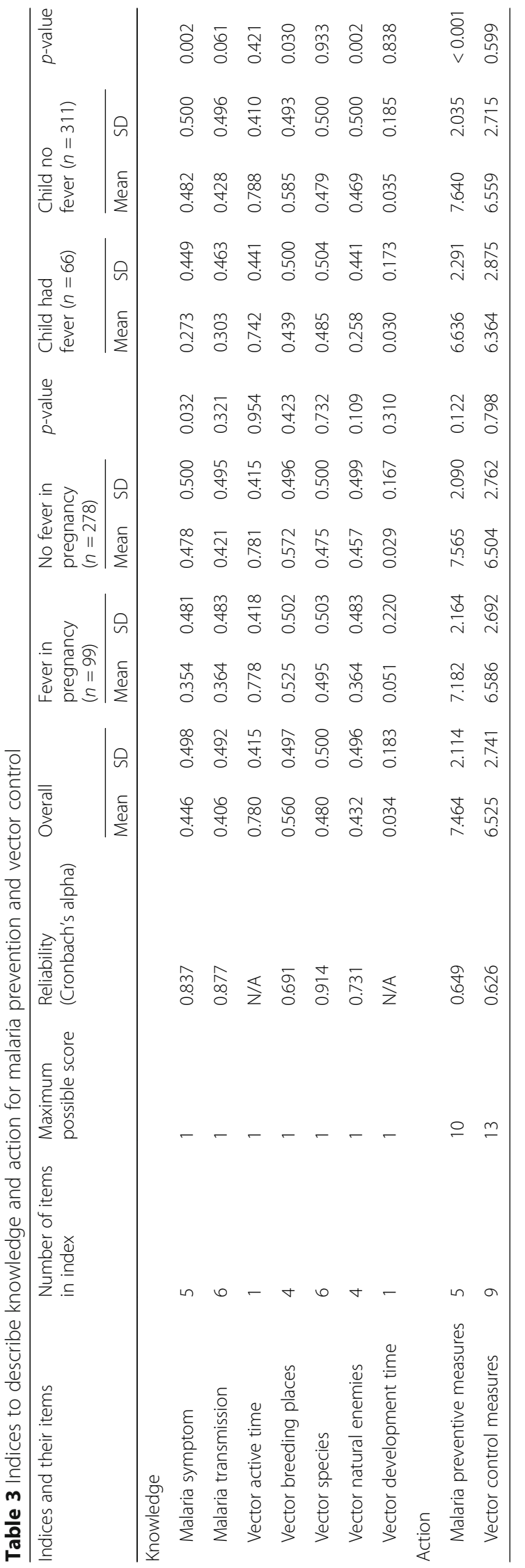


Table 4 Factors associated with the occurrence of fever: during pregnancy and child during the last 3 months

\begin{tabular}{|c|c|c|c|c|c|c|}
\hline \multirow{3}{*}{ Stay overnight at farm hut } & \multicolumn{3}{|c|}{ Multiple logistic regression [Fever in pregnancy] } & \multicolumn{3}{|c|}{ Multiple logistic regression [Fever of child] } \\
\hline & \multirow{2}{*}{$\begin{array}{l}\text { AOR } \\
2.008\end{array}$} & \multicolumn{2}{|l|}{$95 \% \mathrm{Cl}$} & \multirow{2}{*}{$\frac{\mathrm{AOR}}{3.681}$} & \multicolumn{2}{|l|}{$95 \% \mathrm{Cl}$} \\
\hline & & 1.215 & $3.321^{* *}$ & & 1.943 & $6.972^{* *}$ \\
\hline \multicolumn{7}{|c|}{ Malaria preventive measures (ref = least active) } \\
\hline active & 0.980 & 0.518 & 1.853 & 0.497 & 0.234 & 1.056 \\
\hline most active & 0.736 & 0.386 & 1.405 & 0.292 & 0.136 & $0.650^{* *}$ \\
\hline \multicolumn{7}{|c|}{ Vector control measures (ref = least active) } \\
\hline active & 0.888 & 0.485 & 1.626 & 1.297 & 0.623 & 2.702 \\
\hline most active & 1.296 & 0.648 & 2.594 & 2.015 & 0.862 & 4.711 \\
\hline \multicolumn{7}{|l|}{ Age of mother } \\
\hline$<20$ & 1 & & & 1 & & \\
\hline $20-29$ & 0.854 & 0.424 & 1.720 & 0.954 & 0.406 & 2.243 \\
\hline $30-39$ & 0.619 & 0.258 & 1.485 & 0.811 & 0.278 & 2.363 \\
\hline \multicolumn{7}{|l|}{ Education } \\
\hline None & 1 & & & 1 & & \\
\hline Primary & 1.270 & 0.698 & 2.312 & 1.352 & 0.657 & 2.780 \\
\hline Secondary and above & 0.966 & 0.323 & 2.894 & 1.588 & 0.417 & 6.051 \\
\hline \multicolumn{7}{|l|}{ Wealth quartiles } \\
\hline Low & 1 & & & 1 & & \\
\hline Lower middle & 0.709 & 0.322 & 1.560 & 0.874 & 0.343 & 2.233 \\
\hline Upper middle & 0.817 & 0.366 & 1.824 & 0.614 & 0.225 & 1.671 \\
\hline High & 0.574 & 0.229 & 1.440 & 0.803 & 0.272 & 2.374 \\
\hline \multicolumn{7}{|l|}{ Ethnicity } \\
\hline Khmer & 1 & & & 1 & & \\
\hline Thompoun & 1.936 & 0.684 & 5.480 & 4.076 & 0.920 & 18.060 \\
\hline Charay & 0.958 & 0.315 & 2.909 & 3.281 & 0.713 & 15.090 \\
\hline Kreung & 2.158 & 0.751 & 6.199 & 2.112 & 0.455 & 9.809 \\
\hline Other & 1.109 & 0.396 & 3.110 & 2.766 & 0.636 & 12.029 \\
\hline Have other children/sibling (Yes) & 0.885 & 0.476 & 1.646 & 0.935 & 0.434 & 2.016 \\
\hline \multicolumn{7}{|l|}{ Age of child } \\
\hline $0-5$ & - & - & - & 1 & & \\
\hline $6-11$ & - & - & - & 1.456 & 0.701 & 3.024 \\
\hline $12-17$ & - & - & - & 1.154 & 0.512 & 2.601 \\
\hline $18-23$ & - & - & - & 0.713 & 0.285 & 1.783 \\
\hline Gender of child (Female) & - & - & - & 1.317 & 0.725 & 2.394 \\
\hline Experience of child loss (Yes) & - & - & - & 1.923 & 0.832 & 4.460 \\
\hline Water treatment (Yes) & 1.351 & 0.772 & 2.366 & 0.951 & 0.484 & 1.867 \\
\hline Have toilet (Yes) & 1.036 & 0.498 & 2.157 & 1.886 & 0.787 & 4.518 \\
\hline Own livestock (Yes) & 1.445 & 0.738 & 2.829 & 0.939 & 0.419 & 2.107 \\
\hline
\end{tabular}

${ }^{*} p<0.05{ }^{* *} p<0.01$

Major preventive actions against malaria were already undertaken among mothers in our study sites due to continuous efforts by the National Center for Parasitology, Entomology and Malaria Control, Cambodia. For example, the majority of mothers used bed nets at home and wore long-sleeved clothes to protect themselves from malaria. Also, two-thirds of the mothers reported that they brought hammock nets with them when they worked in the forests. However, actions to reduce the number of mosquitoes around their living environments were taken by fewer mothers. More than half of the mothers were not 
Table 5 Treatment-seeking behavior for fever during pregnancy and for children under two

\begin{tabular}{|c|c|c|c|c|}
\hline & \multicolumn{2}{|c|}{$\begin{array}{l}\text { Fever during } \\
\text { pregnancy } \\
(n=99)\end{array}$} & \multicolumn{2}{|c|}{$\begin{array}{l}\text { Children with fever } \\
\text { in the last } 3 \text { months } \\
(n=65)\end{array}$} \\
\hline & $n$ & $\%$ & $n$ & $\%$ \\
\hline \multicolumn{5}{|l|}{ Sought treatment from } \\
\hline Hospital & 2 & 2.0 & 3 & 4.5 \\
\hline Health center & 37 & 37.4 & 31 & 47.0 \\
\hline Private clinic & 5 & 5.1 & 9 & 13.6 \\
\hline Pharmacy & 2 & 2.0 & 6 & 9.1 \\
\hline VMW & 12 & 12.1 & 8 & 12.1 \\
\hline TBA & 2 & 2.0 & - & - \\
\hline Didn't seek treatment & 39 & 39.4 & 9 & 13.6 \\
\hline \multicolumn{3}{|c|}{ Timing of treatment seeking $(n=60)$} & \multicolumn{2}{|c|}{$(n=57)$} \\
\hline Same day & 15 & 26.3 & 18 & 31.6 \\
\hline Next day & 18 & 31.6 & 28 & 49.1 \\
\hline 2 days later & 15 & 26.3 & 7 & 12.3 \\
\hline 3 days or more later & 8 & 14.0 & 4 & 7.0 \\
\hline Don't remember & 4 & 7.0 & - & - \\
\hline \multicolumn{3}{|c|}{ Malaria diagnosis by blood test $(n=60)$} & \multicolumn{2}{|c|}{$(n=57)$} \\
\hline Yes, P.falciparum & 3 & 5.0 & 2 & 3.5 \\
\hline Yes, P.vivax & 2 & 3.5 & 1 & 1.8 \\
\hline Yes, but not sure about result & 10 & 17.5 & 3 & 5.3 \\
\hline Yes, negative & 19 & 33.3 & 18 & 31.6 \\
\hline No, didn't get tested & 10 & 17.5 & 23 & 40.4 \\
\hline Don't remember & 16 & 28.1 & 10 & 17.5 \\
\hline
\end{tabular}

aware of malaria symptoms and its transmission route, and more than two-thirds of the mothers did not know where mosquitoes breed. To ensure malaria elimination, community engagement is the key; elements to increase community engagement include increased knowledge regarding the disease at individual and community levels [18]. Strengthening knowledge about malaria epidemiology and mosquito ecology can boost mothers' actions for malaria prevention and vector control.

Staying overnight at a farm hut was identified as a significant risk factor for developing a fever during pregnancy and for children under two. It has been widely reported that agricultural practices are strongly associated with community health, including malaria incidence [19-21]. Recent studies using PCR diagnosis indicated that an overnight stay in a farm hut is a risk factor for malaria in Ratanakiri Province [5, 6]. Although this study focused on fever episodes, one of the symptoms of malaria, it is possible that malaria risk was increased by mothers' staying overnight at a farm hut. This study provides behavioral and socio-cultural information regarding malaria as it includes data on different aspects of household characteristics and lifestyles, including educational background, ethnicity, wealth, family structure, travel distance to the nearest health centers, malaria knowledge, preventive actions, hygiene practices, and agricultural practices. These factors have not been considered in previous studies. Based on our findings, combined with those from two previous studies, it is strongly recommended that future educational and public health interventions for malaria elimination put high priority on farmers who stay overnight at farm huts in Ratanakiri Province.

In other Asian countries, the association between staying at farm huts and fever or malaria incidence is inconsistent. An entomological and epidemiological study conducted in north-west Thailand reported that malaria infection among farmers engaged in agricultural activities, including movement away from residences to farm huts for ploughing and planting for rice cultivation, was three times that of people living in the residential villages [22]. In Laos, staying overnight in a farm hut was not associated with an increased risk of malaria infection in a rural district, where farmers slept under insecticide-treated bed nets in the huts [23]. Therefore, local malaria epidemiology and a wide variety of socio-demographic and socio-cultural characteristics need to be taken into consideration when examining risk factors for local malaria incidence and/or fever.

We also found that pregnant women and mothers with small children in Ratanakiri Province need to be encouraged to take prompt actions to seek treatment for fevers. A striking finding was that nearly half of the mothers who had fever during pregnancy did not seek treatment. Low use of VMW services could be partly due to temporary suspension of the services in 2015 caused by the delay in payment process (Ly P., personal communication, December 21, 2017). The majority of pregnant woman and children, who received a blood test after running a fever, tested negative for malaria. However, it has been widely reported that pregnant women have an increased susceptibility to infection by Plasmodia spp. [24], and that malaria during pregnancy increases the risk of maternal death, miscarriage, stillbirth, and neonatal death $[25,26]$. Additionally, early diagnosis and effective treatment of malaria is critical for communities to prevent further transmission and spread of the disease [27]. To ensure the elimination of malaria, communities at risk of becoming infected with malaria need to be aware of the importance of treatment-seeking behavior for fever in order to achieve complete and continuous termination of local transmission. Additionally, nonmalarial fevers need either specific treatments or referral to health facilities for appropriate care to reduce maternal and child morbidity and mortality [28]. Therefore, it 
is strongly recommended that future educational interventions stress the importance of treatment-seeking behavior for fevers. Thus, the presence of community health workers such as VMWs in Cambodia, with whom villagers can promptly consult regarding their health issues, is important for malaria elimination.

The limitations of the study must be considered when interpreting these study findings. First, mothers were asked about health issues they experienced and the timing and frequency of their use of health services during their most recent pregnancy, which might have introduced recall bias. It could have resulted in underreporting of health issues and/or inaccurate reports of the number and timing of their health service use. Second, to examine preventive actions and treatment-seeking behavior, mothers' self-reported data were used, which could have introduced courtesy bias. To minimize these biases and improve the accuracy of mothers' self-reports about the timing and frequency of their use of health services, data were confirmed with a maternal health record book and a child health record book whenever available. Moreover, interviews were conducted by experienced and trained interviewers with on-site supervision. Third, sample size or power computation was not possible to be performed because of the lack of information on the number of mothers with children under two. Therefore, recruitment was performed to include as many mothers as possible in the study. However, we conducted a post hoc power analysis, and the sample size was determined to easily achieve a power of greater than $80 \%$ using the effect size we observed.

\section{Conclusions}

To our knowledge, this was the first study to examine malaria knowledge, preventive actions, and treatment-seeking behavior of mothers who belonged to ten ethnic minority groups and the Khmer ethnic group, both of whom reside in remote, agricultural villages in Ratanakiri Province, Cambodia. This study revealed that even though the majority of mothers took malaria preventive measures, knowledge of malaria epidemiology and vector ecology and treatment-seeking behavior for fever were limited. The result indicates the importance of spreading accurate knowledge and promoting prompt treatment-seeking behavior to strengthen community engagement for malaria elimination. Furthermore, staying overnight at a farm hut, regardless of the differences in socio-demographic and socio-cultural characteristics, was strongly associated with fever during pregnancy and childhood. The findings of this study will contribute to the development and implementation of an effective community-based educational program to improve malaria knowledge, raise awareness of health risks related to agricultural practices, and promote treatment-seeking behavior, which will contribute to the achievement of malaria elimination in the region.

\section{Abbreviations}

AOR: adjusted odds ratio; Cl: 95\% confidence interval; CNM: National Center for Parasitology, Entomology, and Malaria Control, Cambodia; MNCH: Maternal, Neonatal, and Child Health; P. falciparum: Plasmodium falciparum; PCR: polymerase chain reaction; VMW: village malaria workers

\section{Acknowledgments}

We are grateful to all mothers who participated in this study as well as all the experts of National Center for Parasitology, Entomology and Malaria Control, National Maternal and Child Health Center, Ratanakiri Provincial Health Department, health centers, and Village Malaria Workers who made this study possible.

\section{Funding}

This study was supported by JSPS KAKENHI Grant Number JP15H05284.

\section{Availability of data and materials}

The datasets used and/or analysed during the current study are available from the corresponding author on reasonable request.

\section{Authors' contributions}

JY conceived the study, developed questionnaire, conducted fieldwork, analysed data, and wrote the manuscript. KK and KN contributed to the study design, conducted fieldwork, and improved the manuscript. PL, BT, and HR supervised fieldwork. TO contributed to the improvement of questionnaire and manuscript. TM monitored the study progress and provided guidance to improve the manuscript. All authors read and approved the final draft.

Ethics approval and consent to participate

Informed consent was obtained from all study participants before conducting the interview. The study protocol, consent forms, and survey questionnaires were approved by the National Ethics Committee for Health Research, the Ministry of Health, Cambodia (368 NECHR), the Research Ethics Committee of Tokyo University of Agriculture and Technology (28-45), and the Research Ethics Committee of the University of Tokyo (11030).

Consent for publication

Written informed consent for publication was obtained.

Competing interests

The authors declare that they have no competing interests.

\section{Publisher's Note}

Springer Nature remains neutral with regard to jurisdictional claims in published maps and institutional affiliations.

\section{Author details}

${ }^{1}$ Research and Education Center for Prevention of Global Infectious Diseases of Animals, Tokyo University of Agriculture and Technology, 3-5-8 Saiwai-cho, Fuchu-shi, Tokyo 183-8508, Japan. ${ }^{2}$ Graduate Education and Research Training Program in Decision Science for Sustainable Society, Kyushu University, Motooka 744, Nishi-ku, Fukuoka-shi, Fukuoka 819-0395, Japan. ${ }^{3}$ Office of International Academic Affairs, Graduate School of Medicine and Faculty of Medicine, The University of Tokyo, 7-3-1 Hongo, Bunkyo-ku, Tokyo 113-0033, Japan. ${ }^{4}$ National center for Parasitology, Entomology \& Malaria Control (CNM), Ministry of Health, \#477 Betong Street (Corner St.92), Village Trapangsvay, Sanakat Phnom Penh Thmey, Khan Sen Sok, Phnom Penh, Cambodia.

Received: 11 July 2018 Accepted: 15 October 2018

Published online: 26 October 2018

\section{References}

1. WHO. Strategy for malaria elimination in the greater Mekong subregion (2015-2030), vol. 1. Geneva: World Health Organization; 2015.

2. Maude RJ, Nguon C, Ly P, Bunkea T, Ngor P, Canavati de la Torre SE, et al. Spatial and temporal epidemiology of clinical malaria in Cambodia 20042013. Malar J. 2014;13:385.

3. CNM. National strategic plan for elimination of malaria in the Kingdom of Cambodia 2011-2015. Phnom Penh: Cambodia National Center for Parasitology Entomology and Malaria Control; 2011. p. 2. 
4. MoH. Cambodia malaria elimination action framework 2016-2020. Phnom Penh: Ministry of Health, Cambodia; 2016. p. 7.

5. Sluydts V, Heng S, Coosemans M, Van Roey K, Gryseels C, Canier L, et al. Spatial clustering and risk factors of malaria infections in Ratanakiri Province, Cambodia. Malar J. 2014;13:387.

6. Kerkhof K, Sluydts V, Heng S, Kim S, Pareyn M, Willen L, et al. Geographical patterns of malaria transmission based on serological markers for falciparum and vivax malaria in Ratanakiri, Cambodia. Malar J. 2016:15:510.

7. Heng S, Durnez L, Mao S, Siv S, Tho S, Mean V, et al. Passive case detection of malaria in Ratanakiri Province (Cambodia) to detect villages at higher risk for malaria. Malar J. 2017:16:104.

8. Tynan A, Atkinson JA, Toaliu H, Taleo G, Fitzgerald L, Whittaker M, et al. Community participation for malaria elimination in Tafea Province, Vanuatu: part II. Social and cultural aspects of treatment-seeking behaviour. Malar J. 2011;10:204.

9. Sharma VR. When to seek health care: a duration analysis for malaria patients in Nepal. Soc Sci Med. 2008:66:2486-94.

10. Kerkhof K, Sluydts V, Willen L, Kim S, Canier L, Heng S, et al. Serological markers to measure recent changes in malaria at population level in Cambodia. Malar J. 2016;15:529.

11. Gryseels C, Peeters Grietens K, Dierickx S, Xuan XN, Uk S, Bannister-Tyrrell M, et al. High mobility and low use of malaria preventive measures among the Jarai male youth along the Cambodia-Vietnam border. Am J Trop Med Hyg. 2015;93:810-8.

12. NIS. Cambodia Demographic and Health Survey 2014. Phnom Penh: National Institute of Statistics, Cambodia, Directorate General for Health, Cambodia, and ICF International; 2015. Available at http://dhsprogram. com/pubs/pdf/FR312/FR312.pdf

13. Yasuoka J, Poudel KC, Poudel-Tandukar K, Nguon C, Ly P, Socheat D, Jimba $M$. Assessing the quality of service of village malaria workers to strengthen community-based malaria control in Cambodia. Malar J. 2010;9:109.

14. Yasuoka J, Poudel KC, Ly P, Nguon C, Socheat D, Jimba M. Scale-up of community-based malaria control can be achieved without degrading community health workers' service quality: the village malaria worker project in Cambodia. Malar J. 2012;11:4.

15. Hasegawa A, Yasuoka J, Ly P, Nguon C, Jimba M. Integrating child health services into malaria control services of village malaria workers in remote Cambodia: service utilization and knowledge of malaria management of caregivers. Malar J. 2013;12:292.

16. Yasuoka J, Mangione TW, Spielman A, Levins R. Impact of education on knowledge, agricultural practices, and community actions for mosquito control and mosquito-borne disease prevention in rice ecosystems in Sri Lanka. Am J Trop Med Hyg. 2006;74:1034-42.

17. Vyas S, Kumaranayake L. Constructing socio-economic status indices: how to use principal components analysis. Health Policy Plan. 2006;21:459-68.

18. Whittaker $M$, Smith $C$. Reimagining malaria: five reasons to strengthen community engagement in the lead up to malaria elimination. Malar J. 2015;14:410

19. Najera JA. Malaria Control: Achievements, Problems and Strategies. Parassitologia. 2001:43:1-89.

20. Hawkes C, Ruel M. The links between agriculture and health: an intersectoral opportunity to improve the health and livelihoods of the poor. Bull World Health Organ. 2006;84:984-90

21. Yasuoka J, Levins R, Mangione TW, Spielman A. Community-based rice ecosystem management for suppressing vector anophelines in Sri Lanka. Trans R Soc Trop Med Hyg. 2006;100:995-1006.

22. Somboon P, Aramrattana A, Lines J, Webber R. Entomological and epidemiological investigations of malaria transmission in relation to population movements in forest areas of north-West Thailand. Southeast Asian J Trop Med Public Health. 1998;29:3-9.

23. Nonaka D, Laimanivong S, Kobayashi J, Chindavonsa K, Kano S, Vanisaveth V, et al. Is staying overnight in a farming hut a risk factor for malaria infection in a setting with insecticide-treated bed nets in rural Laos? Malar J. 2010;9:372.

24. Tegegne B, Getie S, Lemma W, Mohon AN, Pillai DR. Performance of loopmediated isothermal amplification (LAMP) for the diagnosis of malaria among malaria suspected pregnant women in Northwest Ethiopia. Malar J. 2017;16:34.

25. De Beaudrap P, Turyakira E, White LJ, Nabasumba C, Tumwebaze B, Muehlenbachs A, et al. Impact of malaria during pregnancy on pregnancy outcomes in a Ugandan prospective cohort with intensive malaria screening and prompt treatment. Malar J. 2013;12:139.
26. McGready R, Cho T, Keo NK, Thwai KL, Villegas L, Looareesuwan S, et al. Artemisinin antimalarials in pregnancy: a prospective treatment study of 539 episodes of multidrug-resistant plasmodium falciparum. Clin Infect Dis. 2001:33:2009-16.

27. Karyana M, Devine A, Kenangalem E, Burdarm L, Poespoprodjo JR, Vemuri R, et al. Treatment-seeking behaviour and associated costs for malaria in Papua, Indonesia. Malar J. 2016;15:536.

28. D'Acremont V, Kilowoko M, Kyungu E, Philipina S, Sangu W, Kahama-Maro J, et al. Beyond malaria--causes of fever in outpatient Tanzanian children. N Engl J Med. 2014;370:809-17.

\section{Ready to submit your research? Choose BMC and benefit from:}

- fast, convenient online submission

- thorough peer review by experienced researchers in your field

- rapid publication on acceptance

- support for research data, including large and complex data types

- gold Open Access which fosters wider collaboration and increased citations

- maximum visibility for your research: over $100 \mathrm{M}$ website views per year

At BMC, research is always in progress.

Learn more biomedcentral.com/submissions 